1032 STRONG ASSOCIATION BETWEEN PATHOLOGICAL RESPONSE TO NEOADJUVANT CHEMOTHERAPY, TILS AND MODELED CA125 KELIM IN OVARIAN CARCINOMAS: CHIVA TRIAL, GINECO

\begin{abstract}
1,2,3,4 J Pierre-Alexandre* ${ }^{*}, 2 \mathrm{~S}$ Moret, ${ }^{5} \mathrm{O}$ Colomban, ${ }^{3,6} \mathrm{P}$ Combe, ${ }^{3,7} \mathrm{~S}$ Abadie-Lacourtoisie, ${ }^{3,8}$ J Meunier, ${ }^{3,9} \mathrm{~A}$ Floquet, ${ }^{3,10} \mathrm{~L}$ Venat-Bouvet, ${ }^{3,11} \mathrm{C}$ Louvet, ${ }^{3,12} \mathrm{~L}$ Favier, ${ }^{3,13} \mathrm{P}$ Follana, ${ }^{3,14} \mathrm{JP}$ Lotz, ${ }^{3,15} \mathrm{~F}$ Del Piano, ${ }^{3,16} \mathrm{M}$ Leheurteur, ${ }^{3,17} \mathrm{CR}$ Alliot, ${ }^{3,18} \mathrm{G}$ De Rauglaudre, ${ }^{3,19} \mathrm{~N}$ Raban, ${ }^{3,20} \mathrm{~A}$ Chevalier-Place, ${ }^{3,4,21} \mathrm{~A}$ Leary, ${ }^{3,4,5,22} \mathrm{~B}$ You. ${ }^{1}$ Université de Paris, Faculté de médecine, Paris, France; ${ }^{2}$ APHP. Centre, Hopital Cochin, Pathology department, Paris, France; ${ }^{3}$ GINECO, Paris, France; ${ }^{4}$ GINEGEPS, Paris, France; ${ }^{5}$ Université Claude Bernard Lyon 1, EA 3738 CICLY, Lyon, France; ${ }^{6}$ APHP. Centre, Hopital européen Georges Pompidou, Medical Oncology, Paris, France; ${ }^{7}$ Institut de Cancérologie de l'Ouest - ICO - Site Paul Papin, Medical oncology, Angers, France; ${ }^{8}$ Centre Hospitalier Régional d'Orléans, Medical oncology, Orléans, France; 9 Institut Bergonié, Oncology, Bordeaux, France; ${ }^{10}$ Centre Hospitalier Universitaire Dupuytren, Medical oncology, Limoges, France; ${ }^{11}$ Institut Mutualiste Montsouris, Medical oncology, Paris, France; ${ }^{12}$ Centre Georges François Lederc, Medical oncology, Dijon, France; ${ }^{13}$ Centre Antoine Lacassagne, Onco-hematology, Nice, France; ${ }^{14}$ APHP. Sorbonne Université, Hopital Tenon, Medical oncology, Paris, France; ${ }^{15}$ Hôpitaux du Léman, Surgery, Thonon-les-Bains, France; ${ }^{16}$ Centre Henri Becquerel, Medical oncology, Rouen, France; ${ }^{17}$ Centre Hospitalier Alpes Leman, Oncology, Contamine-sur-Arve, France; ${ }^{18}$ Institut Sainte-Catherine, Clinical cancerology, Avignon, France; ${ }^{19} \mathrm{CHU}$ de Poitiers - Pôle Régional de Cancérologie - Hôpital de la Milétrie, Oncology, Poitiers, France; ${ }^{20}$ Centre Oscar Lambret, Gynecology, Lille, France; ${ }^{21}$ Gustave Roussy, Medical oncology, Villejuif, France; ${ }^{22}$ Institut de cancérologie des Hospice Civils de Lyon IC-HCL, Medical oncology, CITOHL, Pierre-Bénite, France
\end{abstract}

\subsection{6/ijgc-2021-ESG0.601}

Introduction/Background* As stated by ESGO-ESMO, there is a need for indicators of chemotherapy efficacy in ovarian carcinoma patients treated in first-line setting (Colombo et al, IGCS, 2020). The pathological chemotherapy response score (CRS) and the modeled CA-125 KELIM during neo-adjuvant chemotherapy were reported as potential markers. Moreover, changes in tumor infiltrating lymphocytes (TILs) after neoadjuvant chemotherapy were reported as a prognostic factor (Leary et al, Cancer Immunol Immunother, 2021). We studied the relationships between changes in TILs, the pathological response (pR) and KELIM in patients treated with neo-adjuvant chemotherapy +/- interval debulking surgery (IDS) from CHIVA phase II trial.

Methodology The patients were enrolled in the randomized phase II trial CHIVA (NCT01583322, neo-adjuvant carboplatin-paclitaxel +/- nintedanib, +/- IDS, $n=188$ patients). KELIM were previously calculated (You et al CCR 2020). The 30 patients with the highest KELIM (very chemosensitive) or the lowest KELIM (poorly chemosensitive) were selected. HEstained sections from available tissue blocks at baseline and after chemotherapy were analyzed for stromal TILs (sTILs, surface of the tumor stroma occupied by lymphocytes) and intra-epithelial TILs (ieTILs, brisk or non-brisk). The pathological response (pR) was assessed on the most tumoral available tissue block obtained after chemotherapy (good response if extensive fibrous changes with no or isolated tumor cells, or $<2 \mathrm{~mm}$ cell clusters). Descriptive statistics assessed the relationships between KELIM, TIL changes, and $\mathrm{pR}$.

Result(s)* No relationships between KELIM and TILs infiltrates on baseline tumor samples were found. However, strong associations were found between KELIM and TIL infiltrates after neo-adjuvant chemotherapy for sTILs (median KELIM for sTILs $0-5 \%$ vs $>5 \%$ : 0.28 versus $1.32, \mathrm{P}<0.001$ ) and for ieTILs (median KELIM for ieTILs non-brisk versus brisk: 0.31 versus $1.31, \mathrm{P}=0.04)$. Similarly, an association was found between KELIM and the quality of $\mathrm{pR}$ (median KELIM for patients with poor vs good $\mathrm{pR}$ : 0.31 versus $1.32, \mathrm{P}=$ $0.05)$.
Conclusion* High consistency was found between the modeled CA125 KELIM calculated during the first 100 days of neoadjuvant chemotherapy and the pathological response, consistent with their values as indicators of the tumor chemosensitivity in first-line setting. Moreover, TILs changes were strongly associated with chemosensitivity, opening hypotheses about the mechanisms of chemosensitivity, and immunotherapy opportunity.

\section{DEVELOPMENT AND VALIDATION OF A MACHINE- LEARNING-DERIVED RNASEQ PROGNOSTIC SIGNATURE IN ENDOMETRIAL CANCER}

\begin{abstract}
${ }^{1,2} \mathrm{G}$ Beinse* ${ }^{*}{ }^{3} \mathrm{MA}$ Le Frere Belda, ${ }^{4,5} \mathrm{~J}$ Pierre-Alexandre, ${ }^{3,5} \mathrm{~N}$ Bekmezian, ${ }^{6} \mathrm{M}$ Koual, ${ }^{7} \mathrm{~S}$ Garinet, ${ }^{5,7}{ }^{1} \mathrm{~K}$ Leroy, ${ }^{8} \mathrm{~N}$ Delanoy, ${ }^{2,7} \mathrm{H}$ Blons, ${ }^{5,8} \mathrm{C}$ Gervais, ${ }^{5,9} \mathrm{C}$ Durdux, ${ }^{5,10} \mathrm{C}$ Chapron, ${ }^{1,5} \mathrm{~F}$ Goldwasser, ${ }^{4,5} \mathrm{~B}$ Terris, ${ }^{3,5} \mathrm{C}$ Badoual, $2,7 \mathrm{P}$ Laurent-Puig, ${ }^{2} \mathrm{~V}$ Taly, ${ }^{2,10} \mathrm{~B}$ Borghese, ${ }^{2,6}$ AS Bats, ${ }^{1,2}$ J Alexandre. 'Institut du Cancer Paris CARPEM, AP-HP, APHP. Centre, Department of medical oncology, Hopital Cochin, PARIS, France; ${ }^{2}$ Centre de Recherche des Cordeliers, "Equipe labélisée Ligue Contre le Cancer ", Sorbonne Université, Université de Paris, INSERM, PARIS, France; Institut du Cancer Paris CARPEM, AP-HP, APHP.Centre, Department of pathology, Hopital Européen Georges Pompidou, Paris, France; ${ }^{4}$ nstitut $d u$ Cancer Paris CARPEM, AP-HP, APHP.Centre, Department of pathology, Hopital Cochin, Université de Paris, PARIS, France; ${ }^{5}$ Université de Paris, Paris, France; ${ }^{6}$ nstitut du Cancer Paris CARPEM, AP-HP, APHP.Centre, Department of gynecological surgery, Hopital Européen Georges Pompidou, Paris, France; 'Institut du Cancer Paris CARPEM, AP-HP, APHP.Centre, Department of Biology, Hopital Européen Georges Pompidou, Paris, France; ${ }^{8}$ Institut du Cancer Paris CARPEM, AP-HP, APHP.Centre, Department of medical oncology, Hopital Européen Georges Pompidou, Paris, France; I Institut du Cancer Paris CARPEM, APHP, APHP.Centre, Department of radiotherapy, Hopital Européen Georges Pompidou, Paris, France; ${ }^{10}$ Institut du Cancer Paris CARPEM, AP-HP, APHP.Centre, Department of gynecological surgery, Hopital Cochin, Paris, France
\end{abstract}

\subsection{6/ijgc-2021-ESG0.602}

Introduction/Background* Because of inter-tumor heterogeneity of endometrial carcinoma (EC), prognostication remains challenging. We aimed to develop a RNAseq signature to stratify EC patient prognosis beyond molecular subtyping.

Methodology A prognostic signature was identified using a LASSO-penalized Cox regression model on TCGA $(\mathrm{N}=543$ patients). A polyA-RNAseq-based method was developed for validation of the signature in a cohort of stage I-IV EC patients treated in two Paris Hospitals between 2010 and 2017. Model performances were evaluated using time-dependent ROC curves (prediction of disease-specific-survival (DSS)). The additional value of the RNAseq signature was evaluated using uni/multivariable Cox models (hazard ratio (HR) with [95\% confidence interval]) and Kaplan-Meier analysis.

Result(s)* Among 209 patients included in the validation cohort (median follow-up 55 months IQR [41-69]), 61 (30\%), $10(5 \%), 52(25 \%)$, and 82 (40\%), had mismatch repair-deficient, POLE-mutated, TP53-mutated tumors, and tumors with no specific molecular profile, respectively. The 38-genes signature accurately predicted DSS (AUC $=80 \%$ ). Using a composite classifier accounting for the RNAseq signature and the TP53mutated group, three groups were identified: good prognosis tumors based on RNAseq signature and without TP53 mutation, characterized by excellent outcome $(\mathrm{N}=103$ patients, 5years DSS rates of 99\%) (reference), poor prognosis tumors based on RNAseq signature and without TP53 mutation $(\mathrm{N}=49$ patients, 5-years DSS rates of $81 \%$; HR: 5.86 [1.16; 29.7]), and TP53-mutated tumors whatever the RNAseq signature $(\mathrm{N}=52$ patients, 5-years DSS rates of 52\%; HR: 11.14 [2.40; 51.7]) (HR adjusted on FIGO stage). In 81 (38\%) patients with adverse features (2020 ESGO/ESTRO/ESP 